Successful closure of PDA was seen in 18/23 (83\%) patients after the following treatment regimens with Paracetamol:

\begin{tabular}{ll} 
Abstract 220 Table 2 & \\
\hline Outcome & Number of patients \\
\hline 1 course Paracetamol & 10 \\
2 courses Paracetamol & 2 \\
1 course Ibuprofen +1 course Paracetamol & 5 \\
2 courses Ibuprofen +1 course paracetamol & 1 \\
\hline
\end{tabular}

Of the remaining 5 patients, outcomes were as follows:

\begin{tabular}{ll} 
Abstract 220 Table 3 & Number of \\
Outcome & 3 \\
\hline $\begin{array}{l}\text { Successful closure of PDA with } 1 \text { course Ibuprofen following } 1 \\
\text { failed course Paracetamol }\end{array}$ \\
$\begin{array}{l}1 \text { failed course of paracetamol, required surgical ligation } \\
2 \text { failed courses paracetamol, } 1 \text { failed course Ibuprofen, required } \\
\text { surgical ligation }\end{array}$ & 1 \\
\hline
\end{tabular}

Conclusions Paracetamol appears to be a safe and effective medical treatment for haemo-dynamically significant PDA in preterm neonates who either have contra-indications to Ibuprofen or where Ibuprofen therapy has failed. The risk of toxicity was low $(0 \%$ in this study) and the closure rate of PDA following treatment was high (83\%). Further studies are needed to determine clinical effectiveness in larger sample sizes and to agree a consensus on how many courses of medical management are appropriate before referring for surgical ligation.

\section{10-YEAR REVIEW OF PATHOGENS AND THEIR ANTIMICROBIAL RESISTANCE PATTERNS AT A TERTIARY NEONATAL INTENSIVE CARE UNIT. DO WE HAVE THE RIGHT ANTIBIOTIC POLICIES?}

Monica Arend-Trujillo, Manjusha Narayanan, Isha Rizal, Naveen Athiraman. UK

\subsection{6/bmjpo-2021-RCPCH.120}

Background The presentation of neonatal sepsis is typically non-specific, and empirical antimicrobial treatment is generally commenced before a causative organism is identified. Using surveillance systems to identify pathogens and their antimicrobial resistance patterns, helps to inform clinicians of what isolates to target and assists them in using antibiotics judiciously. Local antibiotic guideline First line antibiotics:

- Penicillin + Gentamicin.

- Second line: Amoxicillin + Gentamicin + Flucloxacillin.

- Second line where CoNS identified or very likely (e.g., preterm with long line but not systemically 'sick'): Vancomycin

- Third line: Vancomycin and Ceftazidime

\section{Objectives}

- To compile all positive cultures from blood, cerebrospinal fluid and respiratory secretions in a 10 year period

- To identify common causative pathogens in the neonatal intensive care unit.

- To detect susceptibility and resistant patterns to antibiotics recommended in local guideline

Methods Retrospective analysis of data from the microbiology department between 1st April 2010 and 31st March 2020.

Data included all positive blood, cerebrospinal fluid, and respiratory secretions cultures, together with their antimicrobial susceptibility and resistance profile

Results A total of 1642 positive cultures were identified in the 10 -year period, from which $68.4 \%$ came from respiratory secretions, $28.9 \%$ from blood and $2.7 \%$ from cerebrospinal fluid samples.

The five most prevalent organisms in all combined cultures were Coagulase negative staphylococci (CoNS), Staphylococcus aureus, Escherichia coli, Enterobacter species and Klebsiella pneumoniae.

CoNS was the most prevalent organism in blood and cerebrospinal fluid cultures, at $56 \%$ and $34.88 \%$ respectively. GBS was always susceptible to Penicillin and E. coli was resistant to Gentamicin in $5 \%$ of cases.

CoNS was $100 \%$ susceptible to Vancomycin and Linezolid but mostly resistant to Gentamicin and Flucloxacillin.

Conclusions Similar pathogens were seen to national data from other NICUs. No significant difference was seen for most prevalent pathogens in a 10 -year period.

First line antibiotics in our local guideline provided good coverage for early onset sepsis organisms.

Second line antibiotics did not cover CoNS, for patients without central line in situ. However, they provided good cover for all other prevalent pathogens involved in late onset sepsis.

A step up from third line antibiotics is considered, when resistant organisms are suspected or grown, in discussion with the microbiologist.

\section{ACHIEVING SGD 3 GOALS WITH GENETICS, INTERNET TECHNOLOGY AND DATA-DRIVEN IMPROVEMENT}

Liesbeth Siderius, Elmas Citak, Rob Braamburg, Sanath Lamabadururiya, Anton Heys, Marc de Graauw. Netherlands

\subsection{6/bmjpo-2021-RCPCH.121}

Background Some 93 million children under 15 years of age live with a moderate or severe disability (WHO, 2015). Disabilities, such as hearing deficit, cerebral palsy, developmental disabilities, turn out to have genetic causes. In 2019 the WHO launched a classification of digital health interventions v1.0 ${ }^{1}$ : Shared language to describe the uses of digital technology for health. In lower and middle-income countries the use of electronic data in medical care is still on the verge of development. Awareness of available genetic tests could be improved.

Objectives International codes and classification are designed to support data harmonization and data exchange. We questioned how new techniques can promote healthy lives and well-being.

Methods Most states provide maternal and child health handbooks (WHO) containing essential information about 